\title{
TUMOR BUDDING PERITUMORAL ASSESSMENT AS A LYMPH NODE METASTATIC PREDICTION OF PENILE SQUAMOUS CELL CARCINOMA WITH HEMATOXYLIN \& EOSIN STAINING
}

\author{
Marlina Sinaga*, Delyuzar*, Lidya Imelda Laksmi* \\ Department of Anatomical Pathology, Faculty of Medicine, Universitas Sumatera Utara, Medan, Indonesia.*Corresponding Author
}

DOI: 10.29322/IJSRP.11.07.2021.p11580

http://dx.doi.org/10.29322/IJSRP.11.07.2021.p11580

\begin{abstract}
Background: Squamous cell carcinoma (SCC) is the most common malignancy of the penis with mostly lymphogenous metastases to the inguinal lymph nodes. Inguinal lymph node metastasis is the most important factor for predicting the survival of penile SCC patients and determining treatment options. The biological phenomenon of tumor budding has been described in various tumors where it has independent predictive and prognostic relevance. The phenomenon of tumor budding in penile SCC is still unclear.

Objective: To analyze the association of peritumoral tumor budding as a predictor of lymph node metastasis in penile SCC.

Methods: This study was an analytic study with a cross sectional approach on 28 samples of paraffin block patients diagnosed histopathologically as penile SCC. Then, the paraffin blocks were re-cut, followed by staining the slides with H\&E. Peritumoral tumor budding was assessed which was categorized as $<5$ buds (low grade) and 5 buds (high grade). Lymph node metastases were assessed based on the presence of nodes according to the American Joint Committee on Cancer (AJCC) TNM system. The association of peritumoral tumor budding as a predictor of lymph node metastasis in penile SCC was statistically tested.

Results: The highest age was 60-69 years, with a mean age of 51.16 years. The location of the tumor is most often on the glans penis. Most often found in stage IIIB. Most samples with lymph node metastases. LVI was found in 16 cases $(57.1 \%)$, and PNI was found in 6 cases $(21.4 \%)$. The most common peritumoral budding tumor was high grade budding at $60.7 \%$.

Conclusions: The study showed a significant correlation between peritumoral budding tumors and lymph node metastases ( $\mathrm{p}$-value $=0.0001)$ with a prediction that high-grade peritumoral budding tumors had a risk of ten times the risk of lymph node metastasis compared to low-grade peritumoral buds ( $\mathrm{PR}=10.35)$.
\end{abstract}

Keywords: tumor budding, peritumoral, metastases, lymph nodes, penile SCC

\section{INTRODUCTION}

Denile cancer is a rare malignancy, especially in developed countries, but is still a public health and social problem in developing countries. ${ }^{1}$ Squamous cell carcinoma (SCC) is the most common malignancy of the penis with metastases, mostly lymphogenous to the glans gland. regional lymph nodes. Metastasis to the inguinal lymph nodes will determine the patient's prognosis and outcome. ${ }^{2}$

The incidence of penile SCC is most common in developing countries such as Africa, Asia and South America. The incidence of penile SCC is $<1 \%$ of all male malignancies in the United States, with $\pm 2,100$ new cases and \pm 400 deaths annually. ${ }^{3}$ Penile SCC usually affects men between the ages of 50 and 60 years. $^{4}$

As many as $\pm 95 \%$ of all penile cancer cases are penile SCC, followed by various other malignancies such as basal cell carcinoma, penile sarcoma, melanoma, lymphoma and metastatic disease. Penile SCC is treatable if found at an early stage, but has serious physical and psychological consequences. ${ }^{4,5,6}$ Penile SCC has a multifactorial etiology, the most common risk factors being human papillomavirus (HPV) infection, phimosis and poor hygiene, as well as in uncircumcised men, lichen sclerosis and inflammatory conditions such as balanitis xerotica obliterans, precancerous lesions such as Bowen's disease. and Erythroplasia Queyrat, compromised immune system, obesity, smoking, ultraviolet A (UVA) phototherapy, number of sexual partners and socioeconomic status. HPV infection has been associated with penile SCC, for which the mechanisms involved in its pathogenesis have not been fully elucidated. HPV infection has been associated with other malignancies including cervical cancer, anal cancer and oropharyngeal cancer. More than $20 \%$ of patients with penile SCC have tested positive for HPV infection. The prevalence of HPV appears to be much higher in uncircumcised men than in circumcised men. HPV-16 is the type most commonly found in men, followed by HPV-18. The process of penile SCC includes two pathways, namely HPV-related SCC and non-HPV-related SCC. Available data are limited in the literature on HPV-related SCC. ${ }^{4}$ 
Inguinal lymph node metastasis is the most important factor for predicting the survival of penile SCC patients. Lymph node involvement is also important to know because it determines treatment. There are several clinicopathological prognostic factors that can be used to predict lymph node metastasis after penectomy, namely tumor size, degree of tumor differentiation, urethral invasion, lymphovascular invasion and perineural invasion. Determination of histopathological prognostic factors cannot be determined completely from tissue biopsy. ${ }^{2,7}$

The biological phenomenon of tumor budding, namely the growth of tumor cell buds in front of invasive tumors, has been described in various human tumors. Tumor budding has both predictive and prognostic relevance. The phenomenon of tumor budding in penile SCC is still unclear. ${ }^{8}$ Therefore, researchers are interested in investigating the prediction of lymph node metastasis in penile SCC by assessing tumor budding as an independent prognostic factor.

\section{MATERIAL AND METHODS}

\section{Sample selection}

This analytical study with a cross-sectional approach was conducted at the Department of Anatomic Pathology, Faculty of Medicine, University of North Sumatra, Medan and the Anatomic Pathology Unit, H. Adam Malik Hospital, Medan. This research was conducted from June 2020 to May 2021, after obtaining approval from the Health Research Ethics Committee, Faculty of Medicine, University of North Sumatra.

The sample of this study was a paraffin block from patients who had been diagnosed histopathologically as penile SCC that met the inclusion and exclusion criteria. Samples were taken using consecutive sampling technique. Inclusion criteria included adequate clinical data in the medical record (includes age, tumor location, clinical stage and lymph node metastases) as well as representative paraffin slides or blocks, derived from the results of wide excision and penectomy surgery tissue diagnosed histopathologically as penile SCC with staining H\&E. The exclusion criteria for this study were paraffin slides or blocks recorded according to unrepresentative data storage devices so they could not be reprocessed and paraffin slides or blocks derived from excision biopsy tissue results. Each sample was recut and stained with H\&E.

Peritumoral tumor budding is the growth of tumor cell buds in front of an invasive tumor on histopathological preparations with $\mathrm{H} \& \mathrm{E}$ appearance. The assessment of tumor budding refers to a study conducted by Almangush, in which the number of tumor buds was categorized as follows, if $<5$ buds (low grade buds) and $\geq 5$ buds (high grade buds). ${ }^{9}$ Lymph node metastases were assessed based on the presence of nodes referring to the American Joint Committee on Cancer (AJCC) TNM system. ${ }^{10}$ The correlation between the assessment of peritumoral tumor buds as a predictor of lymph node metastasis in penile SCC was tested statistically.

\section{Data analysis}

The correlation between the assessment of peritumoral tumor buds as a predictor of lymph node metastasis in penile SCC was tested statistically.

\section{RESULTS}

In this study, 28 samples of penile SCC were obtained from the Department of Anatomic Pathology, Faculty of Medicine, University of North Sumatra and the Anatomic Pathology Unit, H. Adam Malik Hospital, Medan. The highest age was 60-69 years, with a mean age of 51.16 years. The location of the tumor is most often on the glans penis. Most often found in stage IIIB. Most samples with lymph node metastases. LVI was found in 16 cases $(57.1 \%)$, and PNI was found in 6 cases (21.4\%) (Table 1).

Table 1. Characteristics of study samples based on age, tumor location, clinical stage, lymph node metastases, histopathological subtypes, grade, koilocytes, lymphovascular invasion and perineural invasion in penile SCC patients

\begin{tabular}{|c|c|c|}
\hline Characteristics & $(\mathrm{n}=28)$ & Persentase (\%) \\
\hline \multicolumn{3}{|l|}{ Age (years old): } \\
\hline $20-29$ & 2 & 7,1 \\
\hline $30-39$ & 4 & 14,3 \\
\hline $40-49$ & 7 & 25,0 \\
\hline $50-59$ & 6 & 21,4 \\
\hline $60-69$ & 9 & 32,2 \\
\hline \multicolumn{3}{|l|}{ Tumor location: } \\
\hline Glans penis & 11 & 39,3 \\
\hline Preputium & 1 & 3,6 \\
\hline Coronal sulcus & 0 & \\
\hline Corpus penile & 1 & 3,6 \\
\hline More than one location & 6 & 21,4 \\
\hline \multirow{2}{*}{\multicolumn{3}{|c|}{$\begin{array}{l}\text { No data } \\
\text { Clinical stage: }\end{array}$}} \\
\hline & & \\
\hline Stage I & 5 & 17,9 \\
\hline Stagere II & 6 & 21,4 \\
\hline Stage IIIA & 5 & 17,9 \\
\hline Stage IIIB & 9 & 32,1 \\
\hline \multirow{2}{*}{\multicolumn{3}{|c|}{ Lymph node metastages: }} \\
\hline & & \\
\hline No & 11 & 39,3 \\
\hline Present & 17 & 60,7 \\
\hline \multicolumn{3}{|l|}{ Histopathology subtype } \\
\hline SCC, usual type & 3 & 10,71 \\
\hline Basaloid carcinoma & 9 & 32,15 \\
\hline Warty carcinoma & 13 & 46,43 \\
\hline \multirow{2}{*}{\multicolumn{3}{|c|}{ 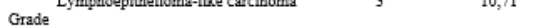 }} \\
\hline & & \\
\hline Grade I & 5 & $\begin{array}{r}17,85 \\
53,58\end{array}$ \\
\hline $\begin{array}{l}\text { Grade II } \\
\text { Grade III }\end{array}$ & $\begin{array}{c}15 \\
8\end{array}$ & $\begin{array}{l}53,58 \\
28,57\end{array}$ \\
\hline \multicolumn{3}{|l|}{$\begin{array}{l}\text { Coilocyte } \\
\text { Cll II }\end{array}$} \\
\hline No & 3 & 10,71 \\
\hline Present & 25 & 89,29 \\
\hline \multicolumn{3}{|l|}{ Lxomphovascular invagion } \\
\hline No & 12 & 42,9 \\
\hline $\begin{array}{c}\text { Present } \\
\text { Perineural intragion }\end{array}$ & Perineural invasion & 57,1 \\
\hline $\begin{array}{l}\text { Perineural invasion } \\
\text { No }\end{array}$ & 22 & 78,6 \\
\hline Present & 6 & 21,4 \\
\hline
\end{tabular}
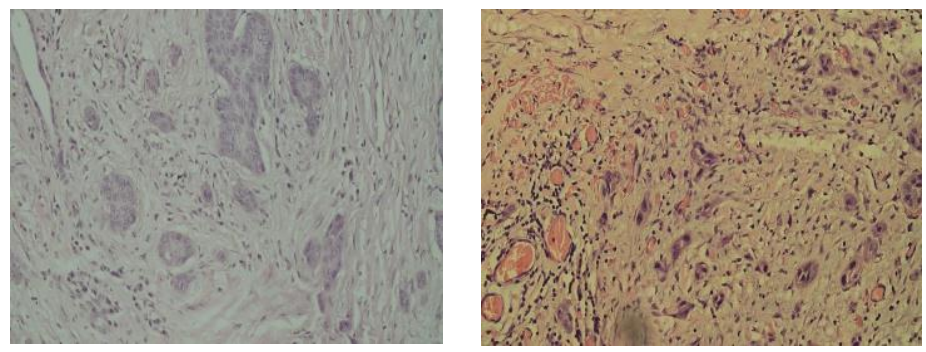

Figure 1. Low grade buds (H\&E, X200). B. High grade buds (H\&E, X200).

In this study, the association between peritumoral budding tumors and lymph node metastases in patients with penile SCC was assessed. The most common peritumoral budding tumor was high grade budding at $60.7 \%$. The study showed a significant 
correlation between peritumoral budding tumors and lymph node metastases $(p$-value $=0.0001$ ) with a prediction that high-grade peritumoral budding tumors had a risk of ten times the risk of lymph node metastasis compared to low-grade peritumoral buds $(\mathrm{PR}=10.35)$ (Table 2).

Table 2. Correlation between peritumoral budding tumors and lymph node metastases in patients with penile SCC.

\begin{tabular}{|c|c|c|c|c|c|c|c|}
\hline \multirow{3}{*}{$\begin{array}{c}\text { Tumor Budding } \\
\text { Peritumoral }\end{array}$} & \multicolumn{4}{|c|}{ Lymph Node Metastases } & \multirow{3}{*}{$P R$} & \multirow{3}{*}{$95 \% \mathrm{CI}$} & \multirow{3}{*}{ P-value } \\
\hline & \multicolumn{2}{|c|}{$(+)$} & \multicolumn{2}{|c|}{$(-)$} & & & \\
\hline & $\mathrm{N}$ & $\%$ & $\mathrm{~N}$ & $\%$ & & & \\
\hline Low grade buds & 1 & 9,1 & 10 & 90,9 & & & \\
\hline High grade buds & 16 & 94,1 & 1 & 5,9 & 10,35 & $1,59-67,34$ & 0,0001 \\
\hline Total & 17 & 60,7 & 11 & 39,3 & & & \\
\hline
\end{tabular}

\section{DISCUSSION}

The number of samples diagnosed as penile SCC in this study were 28 samples, of which 9 patients were aged 60-69 years, with a mean of 51.16 years, where the youngest age was 26 years and the oldest was 67 years. The results of this study are not much different from previous studies. De-Bacco et al. in 2019 found that the mean age of the patients was 64.6 years with a standard deviation of 12.4 years.11 Douglawi et al. in 2017 stated that penile SCC mainly affects men between the ages of 50 and 70 years. ${ }^{12}$ Wang et al. in 2019 stated that the average penile SCC patient was 64 years old with a range of $27-86$ years. ${ }^{13}$ The discovery of young patients proves that at this time there are many younger penis SCC patients. Penile SCC patients who are young, can be caused by multifactorial causes, one of which is strongly suspected due to an unhealthy lifestyle. Such as eating a lot of unhealthy foods, rarely exercising, smoking and other bad habits. Plus most young people sometimes don't pay attention to their health. They tend to be indifferent and lazy to maintain and check their health condition.

In this study, the location of the most tumors was on the glans penis $(39.3 \%)$, then successively at more than one location $(21.4 \%)$, where the tumor locations could be found simultaneously, either in the radix, body and or glans. penis and the least is a tumor located in the prepuce (3.6\%) which is a refractile fold of skin on the glans penis and body of the penis $(3.6 \%)$. However, of the total samples examined, there were 9 patients with unclear locations, this was due to the fact that the location of the tumor was not recorded in the medical record. The results of this study are not much different from previous studies. Greenberg in 2010 reported that the location of the most tumors on the glans penis, then the coronal sulcus and the inner surface of the prepuce, respectively. ${ }^{14}$ Chaux et al. in 2010 reported that the location of the most tumors on the glans penis. ${ }^{15}$ However, contrary to Lagace et al. in 2020 reported that the most tumor locations were in unspecified areas $(54 \%)$, then the glans penis $(25 \%)$, prepuce $(16 \%)$, body of the penis $(3 \%)$, more than one lesion $(2 \%)$ respectively. ${ }^{16}$ Most penile SCC in uncircumcised men initially occurs in the prepuce and in circumcised men it initially occurs in the glans penis. Penile SCC, if found at an early stage, is usually curable. ${ }^{17}$ This discrepancy in results suggests that differences in the specific location of penile SCC are associated with patient survival. Tyson et al. in 2012 reported his study conducted on 2,515 cases of penile SCC over a period of 34 years, stating that tumors in the body of the penis and in more than one location have a higher increased risk of death compared to tumors in the prepuce. Long-term survival was significantly worse for patients with tumors involving areas other than the foreskin of the penis. In contrast, patients with penile SCC located in the prepuce have better long-term survival. Based on the results of this study, they have potentially important clinical implications when assessing the prognosis of patients diagnosed as penile SCC. ${ }^{18}$

In this study, there were 9 patients with penile SCC with stage IIIB. This seems to be due to the fact that penile SCC is still a public health and social problem in developing countries. Penile SCC is usually associated with an inadequate health care system, poor hygiene, high rates of sexually transmitted infections and phimosis. Delay in diagnosis and treatment of patients is associated with lower socioeconomic status and less education. So this will result in patients usually coming already in an advanced stage condition

In this study, there were 17 patients who had metastases to the lymph nodes. This is in accordance with the stage found in this study which was also found to be mostly at stage IIIB. Sali et al. in 2019 stated that inguinal lymph node metastasis is the most important factor for predicting the survival of penile SCC patients. Lymph node involvement is also important to know because it determines treatment. 5-year survival dropped dramatically from $95.7 \%$ to $51.1 \%$ with inguinal lymph node involvement. ${ }^{1} \mathrm{Hu}$ et al. in 2019 stated that higher-stage penile SCC had a higher risk of lymph node metastasis. The risk of lymph node metastasis will increase significantly as long as there are invasive pathological characters, such as lymphovascular invasion, corpora cavernosum invasion, corpus spongiosum invasion, urethral invasion, perineural invasion, larger tumor size, and deeper tumor invasion. ${ }^{19}$

Based on the pathological classification of penile SCC according to WHO in 2016, penile SCC consists of eleven histopathological subtypes, of which eight histopathological subtypes of Non-HPV related and three histopathological subtypes of HPV-related. In this study, the researchers assessed the histopathological subtypes based on histomorphological features with $H \& E$ staining and the presence of koilocyte cells in the study sample. So the researchers only found four histopathological subtypes of all histopathological subtypes in penile SCC, the majority were warty carcinoma subtypes, namely 13 samples (46.43\%), and there were three other histopathological subtypes, namely basaloid carcinoma, SCC, usual type and lymphoepithelioma-like carcinoma. Afonso et al in 2012 reported that $30-70 \%$ of cases of penile SCC were HPVrelated. ${ }^{20}$ The difference in results in this study could be attributed possibly to differences in the study population as well as risk factors such as multiple sexual partners and low public awareness. in terms of condom use, where this affects the high incidence of HPV infection. It is important for a pathologist to continue to assess the histopathological subtypes of penile SCC based on the 2016 WHO classification of penile SCC, which will be divided into Non-HPV related and HPV related. Although PCR examination is the gold standard for HPV detection. These 
histopathological subtypes differ in terms of histomorphological features and patient outcomes.

In this study there were as many as $53.58 \%$ of the penis SCC samples were grade II. The results of this study are not much different from previous studies. Sali et al. in 2019 found that the most common grade II (moderately differentiated carcinoma) cases were 59\%. ${ }^{1}$ Zheng et al. in 2020 found that the most common grade II (moderately differentiated carcinoma) cases were $57.3 \% .^{21} \mathrm{Nam}$ et al. in 2017 reported that the most cases of well to moderately differentiated carcinoma were found at $82.7 \%$. Penile SCC accounts for $>95 \%$ of cases of penile malignancy. Penile SCC has been described as a locoregional disease with a low incidence of distant metastases. So that most penile tumors are classified as low grade. ${ }^{22}$

In this study as many as $82.29 \%$ of all samples of penile SCC found the presence of koilocytes. In a study conducted by Drummond et al. in 2019 reported that as many as $41.8 \%$ of the total samples studied found the presence of koilocytes. The difference in the results of this study can be attributed possibly to differences in the study population. The incidence of penile SCC is related to sexual habits and promiscuity. Habit factors where the lack of condom use has a major influence on the incidence of HPV infection. ${ }^{23} \mathrm{HPV}$ is a non-enveloped DNA virus with circular double-stranded DNA. After infection of the basal cells, HPV transcribes one of its two strands of DNA, thereby leading to the formation of viral proteins. Two proteins, E6 and E7, are responsible for the oncogenic effects of the virus. E6 and E7 interfere with the apoptotic pathway and promote cell proliferation by interfering with the tumor suppressor genes RB and p53. If the virus inserts its DNA into the genome, it will replicate in mature squamous cells. The koilocytic morphology results from the E4 protein, which acts to disrupt the squamous cell cytoskeleton. ${ }^{24}$ Krawczyk et al. stated that HPV E5 and E6 proteins work together to promote the formation and fusion of perinuclear cavitations. This fusion is likely to create large cavitation that distinguishes HPV-infected cells with clear cell images around the cell nucleus. ${ }^{25}$ Assessment of the presence of koilocytes is important to assess, where these koilocytes also help in determining the histopathological subtype of HPV-related penile SCC, so that the presence of these koilocytes will affect the outcome of the patient. The presence of koilocytes in the subtype of warty carcinoma has a lower mortality rate than the subtypes of basaloid carcinoma and lymphoepithelioma-like carcinoma.

In this study, lymphovascular invasion was found in $57.1 \%$. The remaining 12 samples did not find lymphovascular invasion (42.9\%). In a study conducted by $\mathrm{Li}$ et al. in 2019 who found lymphovascular invasion as much as $20.9 \% .{ }^{26}$ Lymphovascular invasion is defined as the presence of tumor cell invasion into the blood vessels or lymphatic system. ${ }^{27}$ Lymphovascular invasion is the primary and crucial phase in systemic metastasis of cancer cells. $^{28}$ There is increasing evidence to show that that the presence of lymphovascular invasion is a poor prognostic indicator in various malignancies, including bladder cancer. ${ }^{29}$ Liu et al. in 2013 reported that lymphovascular invasion was not significantly associated with the overall survival of patients with penile SCC. ${ }^{30}$ The differences in the results of this study could be attributed possibly to differences in the study population.
In this study, perineural invasion was reported in $21.4 \%$. In a study conducted by Jain in 2017 which found $19 \%$ perineural invasion. ${ }^{8}$ Sali et al. in 2019 stated that perineural invasion was significantly associated with the presence of lymph node metastases with $\mathrm{p}<0.001 .^{1}$ Marie et al. in 2020 stated that the presence of perineural invasion is a poor prognostic indicator. In multivariate analysis, perineural invasion was associated with local recurrence of the disease with $\mathrm{p}$-value $=0.02 .{ }^{31}$ Perineural invasion was used to describe the process of invasion into, around and through nerves. ${ }^{32}$ Perineural invasion in cancer is also defined as tumors near nerves and involving at least $33 \%$ of the circumference or tumor cells were within one of the three neural sheaths. ${ }^{33}$ There is considerable variability in the reported incidence of perineural invasion in penile SCC possibly due to variability of the subtype and stage of penile SCC.

In this study, the most common peritumoral budding tumors were high grade buds as much as $60.7 \%$ compared to low grade buds only as much as $39.3 \%$. From the analysis of the correlation between peritumoral budding tumors and lymph node metastases, it was found that the $\mathrm{p}$-value $=0.0001$, it can be concluded that there is a significant correlation between peritumoral budding tumors and lymph node metastasis. The results of the statistical test also obtained a PR value of 10.35 , so it can be concluded that the findings of high grade peritumoral budding tumors have a risk of lymph node metastasis ten times compared to low grade peritumoral budding tumors. From the results of statistical tests in this study, it can be concluded that the assessment of peritumoral budding tumors can be used as a prediction of lymph node metastasis in penile SCC. The results of this study are supported by Chatterjee et al. in 2019 which stated that there was a significant correlation between tumor budding in oral SCC and lymph node metastasis. ${ }^{34}$ Almangush et al. in 2013 reported that tumor budding independently predicts survival in oral tongue SCC patients. Tumor budding has been associated with patient prognosis in various types of epithelial cancer, such as esophageal, lung, colorectal and endometrial carcinoma. ${ }^{9}$ Meanwhile, Jain in 2017 stated that there was no significant correlation between tumor budding and lymph node metastasis. The difference from this study lies in the scoring system used for tumor budding. The number of buds found was divided into three frequency groups: less than 10 buds, $10-20$ buds and more than 20 buds. After changing the shoot class division into class $1(\leq 15$ buds) and class 2 (>15 buds), it was observed in the KaplanMeier analysis that patients with more than 15 buds had a slightly worse prognosis than patients with 15 or fewer tumors. budding. ${ }^{8}$ The difference in the results of this study may be due to differences in the assessment system for the number of budding tumors used.

The limitation of the study is that there is no uniform cut-off value for the number of budding tumors in penile SCC. Therefore, the researcher used a tumor budding number grading system based on a study by Almangush et al. used for oral tongue SCC. The results of the tumor budding assessment were divided into two categories, namely if $<5$ buds were referred to as low grade buds and if 5 buds were referred to as high grade buds.

\section{CONCLUSION}


After conducted this study, we conclude some points in the following:

1. Most patients with penile SCC occur at the age of 60-69 years with the mean age of patients being 51.16 years, where the youngest age is 26 years and the oldest age is 67 years. The location of the most tumors on the glans penis. Most of the clinical stages were in stage IIIB, and most of the research samples were found to have metastasized to lymph nodes.

2. The most common peritumoral budding tumors are high grade buds. There is a significant correlation between peritumoral budding tumors and lymph node metastasis. Peritumoral budding tumors with high grade buds had a ten times risk of lymph node metastasis compared to peritumoral budding tumors with low grade buds.

3. The most histopathological subtypes were warty carcinoma, followed by basaloid carcinoma, SCC, usual type and lymphoepithelioma-like carcinoma. Most grades are in grade II, followed by grade III. The least is in grade I. The presence of koilocytes is $89.29 \%$. Lymphovascular invasion was $57.1 \%$. Perineural invasion was $21.4 \%$.

\section{COMPETING INTERESTS}

The author has no financial interests relevant to the product or company described in this article.

\section{ACKNOWLEDGMENT}

We would like to thank all staff of the Department of Anatomical Pathology, University of North Sumatra, Private hospitals and private clinics in Medan, Indonesia for all their assistance and cooperation.

\section{ETHICAL APPROVAL}

Health Research Ethical Committee, Universitas Sumatera Utara, Medan, Indonesia approved this study.

\section{REFERENCES}

[1] Sali AP, Menon S, Prakash G, Murthy V, Bakshi G, Mahantshetty U, et al. Histopathological risk scoring system as a tool for predicting lymph nodal metastasis in penile squamous cell carcinoma. 2019;51(7):696-704.

[2] Lestari D, Mulyadi K, Moestikaningsih. Hubungan antara Ukuran Tumor, Grade, Invasi Uretra, Intravasa, Perineural dan Overekspresi COX-2 pada Metastasis Karsinoma Sel Skuamosa Penis ke Kelenjar Getah Bening Inguinal. Majalah patologi. 2015;24(3):34-40

[3] Siegel RL, Miller KD, Jemal A. Cancer statistics, 2019. CA Cancer J Clin. 2019;69:7-34.

[4] Iorga L, Marcu R, Diaconu C, Stanescu A, Stoian A, Mischianu D, et al. Penile carcinoma and HPV infection (Review). Exp Ther Med. 2019:1-6.

[5] Brierley JD, Gospodarowicz MK, Wittelind C, editors. TNM classification of malignant tumours, ed. 8. Chichester, UK. Wiley \& Son. 2017.

[6] Sanchez DF, Soares F, Alvarado CI, Cañete S, Fernández MJ, Rodríguez IM, et al. Pathological factors, behavior, and histological prognostic risk groups in subtypes of penile squamous cell carcinomas (SCC). Semin Diagn Pathol. 2015;32:222-231.

[7] Erbersdobler A. Pathology and histopathological evaluation of penile cancer. Urologe. 2018;57:391-397.
[8] Jain A. Retrospektive morphologische Untersuchungen zum TumorBudding bei Plattenepithelkarzinomen des Penis. German. 2017:1-156.

[9] Almangush A, Pirinen M, Heikkinen I, Makitie AA, Salo T, Leivo I. Tumour budding in oral squamous cell carcinoma: a meta-analysis. British Journal of Cancer. 2018;118:577-586.

[10] Cubilla AL, Amin MB, Ayala A, Chaux A, Corbishley C, Dillner J, et al. Malignant epithelial tumours In: Holger M, Peter A, Thomas M, Victor E, eds. WHO Classification of Tumours of the Urinary System and Male Genital Organs; $4^{\text {th }}$ Ed. 2016. Lyon:IARC. 2016:262-276.

[11] De-Bacco MW, Carvalhal GF, MacGregor B, Marçal JMB, Wagner MB, Sonpavde GP, et al. PD-L1 and p16 Expression in Penile Squamous Cell Carcinoma From an Endemic Region. Clin Genitourin Cancer. 2020;18(3):e254-e259. doi: 10.1016/j.clgc.2019.10.014

[12] Douglawi A, Masterson T. Updates on the epidemiology and risk factors for penile cancer. Transl Androl Urol. 2017;6:785-790.

[13] Wang Y, Wang K, Chen Y, Zhou J, Liang Y, Yang X, et al. Mutational landscape of penile squamous cell carcinoma in a Chinese population. Int $\mathbf{J}$ Cancer. 2019;145(5):1280-1289. doi: 10.1002/ijc.32373

[14] Greenberg RE. Surgical management of carcinoma of the penis. Urol Clin North Am. 2010;37:369-378.

[15] Chaux A, Pfannl R, Lloveras B, Alejo M, Clavero O, Lezcano C, et al. Distinctive association of p16INK4a overexpression with penile intraepithelial neoplasia depicting warty and/or basaloid features: a study of 141 cases evaluating a new nomenclature. Am J Surg Pathol. 2010;34(3):385-392. doi: 10.1097/PAS.0b013e3181cdad23

[16] Lagacé F, Ghazawi FM, Le M, Savin E, Zubarev A, Powell M, et al. Penile Invasive Squamous Cell Carcinoma: Analysis of Incidence, Mortality Trends, and Geographic Distribution in Canada. J Cutan Med Surg. 2020;24(2):124-128. doi: 10.1177/1203475419888869

[17] What Is Penile Cancer? [Internet]. American Cancer Society. 2018 [cited 6 April 2021]. Available from: https:/www.cancer.org/cancer/penilecancer/about/what-is-penile-cancer.html

[18] Tyson MD, Etzioni DA, Wisenbaugh ES, Andrews PE, Humphreys MR, Ferrigni RG, et al. Anatomic site-specific disparities in survival outcomes for penile squamous cell carcinoma. Urology. 2012;79(4):804-808. doi: 10.1016/j.urology.2011.12.047

[19] Hu J, Cui Y, Liu P, Zhou X, Ren W, Chen J, et al. Predictors of inguinal lymph node metastasis in penile cancer patients: a meta-analysis of retrospective studies. Cancer Management and Research 2019;11:64256441.

[20] Afonso LA, Moyses N, Alves G, Ornellas AA, Passos MRL, Oliveira LHS, et al. Prevalence of human papillomavirus and Epstein-Barr virus DNA in penile cancer cases from Brazil. Memórias do Instituto Oswaldo Cruz. 2012;10:18-23

[21] Zheng W, Li K, Zhu W, Ding Y, Wu Qingna, Tang Qiling, et al. Nomogram prediction of overall survival based on log odds of positive lymph nodes for patients with penile squamous cell carcinoma. Cancer Med. 2020;9:5425-5435.

[22] Nam JK, Lee DH, Park SW, Kam SC, Lee KS, Kim TH, et al. Clinicopathologic Characteristics and Treatment Outcomes of Penile Cancer. World J Mens Health. 2017;35:29-33.

[23] Drummond M, Lage S, Salomao L, Junior EP, Salles PG, Salazar AL, et al. Epidemiological Aspects of Penile Cancer in Minas Gerais: Evaluation of 55 Cases from Two Referring Hospitals. Uronimas. 2019:36-41.

[24] Krause KA, Neelon D, Butler SL. Koilocytosis. StatPearls [Internet]. Treasure Island (FL). 2021 [cited 23 April 2021]. Available from: https://www.ncbi.nlm.nih.gov/books/NBK532958/

[25] Krawczyk E, Suprynowicz FA, Liu X, Dai Y, Hartmann DP, Hanover J, et al. Koilocytosis: a cooperative interaction between the human papillomavirus E5 and E6 oncoproteins. Am J Pathol. 2008;173(3):682-688.

[26] Li K, Sun J, Wei, X, Wu G, Wang F, Fan C, et al. Prognostic value of lymphovascular invasion in patients with squamous cell carcinoma of the penis following surgery. BMC Cancer. 2019:476(19);1-11. https://doi.org/10.1186/s12885-019-5714-1

[27] Hamy A, Lam G, Laas E, Darrigues L, Balezeau T, Guerin J, et al Lymphovascular invasion after neoadjuvant chemotherapy is strongly associated with poor prognosis in breast carcinoma. Breast Cancer Res Tr. 2018;169:295 -304.

[28] Shariat SF, Svatek RS, Tilki D, Skinner E, Karakiewicz PI, Capitanio U, et al. International validation of the prognostic value of lymphovascular invasion in patients treated with radical cystectomy. BJU Int. 2010;105:1402-1412. 
[29] Mathieu R, Lucca I, Rouprêt M, Briganti A, Shariat SF. The prognostic role of lymphovascular invasion in urothelial carcinoma of the bladder. Nat Rev Urol. 2016;13:471-479.

[30] Liu J, Li Y, Zhang Z, Yao K, Ye Y, Xie D, et al. The risk factors for the presence of pelvic lymph node metastasis in penile squamous cell carcinoma patients with inguinal lymph node dissection. World J Urol. 2013;31:1519-1524

[31] Marie-Lisa E, Maria-del CRP, Schwartz L, Carlos PG, Rais-Bahrami S, Giannico G, et al. Morphology, p16, HPV, and outcomes in squamous cell carcinoma of the penis: a multi-institutional study. Hum Pathol. 2020;96:79-86.

[32] Alkhadar H, Macluskey M, White S, Ellis I. Perineural invasion in oral squamous cell carcinoma: Incidence, prognostic impact and molecular insight. J Oral Pathol Med. 2020;49(10):994-1003. doi: 10.1111/jop.13069. Epub 2020 Jun 25

[33] Liebig C, Ayala G, Wilks JA, Berger DH, Albo D. Perineural invasion in cancer: a review of the literature. Cancer. 2009;115(15):3379-3391.

[34] Chatterjee D, Bansal V, Malik V, Bhagat R, Punia RS, Handa U, et al. Tumor Budding and Worse Pattern of Invasion Can Predict Nodal Metastasis in Oral Cancers and Associated With Poor Survival in EarlyStage Tumors. Ear, Nose \& Throat Journal. 2019;98(7):E112-E119.

\section{AUTHORS}

First author- dr. Marlina Sinaga, Resident of the Department of Anatomical Pathology, Faculty of Medicine, Universitas Sumatera Utara, Medan, Indonesia, email ID: dr.marlinasinaga82@gmail.com.

Second Author- Dr. dr. Delyuzar, M.Ked (PA) Sp.PA (K). Department of Anatomical Pathology, Faculty of Medicine, Universitas Sumatera Utara, Medan, Indonesia.

Third author- Dr. dr. Lidya Imelda Laksmi, M.Ked (PA), Sp.PA, Department of Anatomical Pathology, Faculty of Medicine, Universitas Sumatera Utara, Medan, Indonesia.

Correspondence Author- Dr. dr. Lidya Imelda Laksmi, M.Ked (PA) Sp.PA Department of Anatomical Pathology, Faculty of Medicine, Universitas Sumatera Utara, Medan, Indonesia, email ID:lidyaimelda76@gmail.com 